\title{
El corte de rocas ornamentales con discos diamantados: influencia de los factores propios del sistema de corte
}

Fecha de recepción:20-X-97

Fecha de aceptación: 15-I-98

\author{
L. M. SUÁREZ DEL RÍ, A. RODRÍGUEZ-REY, L. CALLEJA y V. G. RUIZ DE ARGANDOÑA
Dpto. de Geología. Área de Petrología y Geoquímica. Univ. de Oviedo \\ L. M. SUÁREZ DEL RÍ, A. RODRÍGUEZ-REY, L. CALLEJA y V. G. RUIZ DE ARGANDOÑA
Dpto. de Geología. Área de Petrología y Geoquímica. Univ. de Oviedo
}

ESPAÑA

\section{RESUMEN}

En este trabajo se hace una revisión de algunas publicaciones relevantes relacionadas con el corte de rocas ornamentales con discos diamantados, con el propósito de que pueda ser de utilidad a los industriales del Sector de la Piedra Natural. Debido a la extensión del trabajo, se ha dividido en dos partes: en esta primera se analiza la influencia de los factores propios del sistema de corte (maquinaria, discos, características del corte, etc.), y en la segunda parte, en un próximo número de esta revista, se hará hincapié en las características propias de la roca que influyen en su cortabilidad y, por tanto, en el rendimiento de los útiles diamantados.

\section{SUMMARY}

A review of some relevant papers about the cutting of ornamental stones with diamond tools is presented here with the aim of being useful to the Natural Stone industries. Due to the length of this paper, it has been divided in two parts: in the first one, the influence of the factors of the cutting equipments (machinary, disks, cutting characteristics, etc) is reviewed, and in the second one, in the next number of this journal, attention will be paid to the intrinsic rock characteristics that influences its cuttability and, thus, the eficiency of the diamond tools.

\section{INTRODUCCIÓN}

La incorporación del diamante a los procesos de extracción y elaboración de rocas ornamentales ha contribuido de forma fundamental al cambio tecnológico experimentado en los últimos 35 años en el sector de la Piedra Natural. La implantación del corte con útiles diamantados (discos, hilos y telares) e incluso del desbaste y pulido con diamante, ha hecho que en la actualidad la mayor parte de las rocas ornamentales del mundo hayan "estado en contacto" con el diamante en alguna fase de su producción.

El estudio de la capacidad de corte de las rocas con útiles diamantados, sobre todo discos, es un tema al que se le ha prestado atención desde hace bastante tiempo. No hay duda que tiene una clara incidencia práctica especialmente en el sector de la Piedra Natural, ya que, en el cálculo de los costes totales, la vida útil de los discos es un factor importante a considerar. Estos estudios han perseguido, entre otras cosas, mejorar el rendimiento del corte aplicando las condiciones óptimas a cada roca en particular.

En el corte con útiles diamantados se producen dos fenómenos recíprocos: el útil diamantado corta la roca pero, a su vez, la roca desgasta el útil. Por eso, el estudio puede llevarse a cabo desde dos puntos de vista: uno, intentando determinar la influencia de las características de las rocas en su cortabilidad, y otro, estudiando las condiciones óptimas del útil y del procedimiento de corte que permitan un aprovechamiento más eficiente, sin olvidar 
tampoco las características técnicas del equipo o máquina de corte.

Es evidente que es imposible separar del todo ambos aspectos, aunque los estudios pueden tener un enfoque distinto, dependiendo de cuál de ellos se considere prioritario.

Así pues, en el proceso de corte de rocas intervienen conjuntamente el equipo o sierra de corte, el útil diamantado y el material a cortar. Además, no se deben olvidar los parámetros o condiciones del corte ni quizás el factor más importante: el humano.

A continuación se expondrán algunas consideraciones respecto a la influencia de los factores extrínsecos (ajenos al material rocoso a cortar), referidos fundamentalmente al corte con discos diamantados, aunque algunos aspectos serían comunes al corte con telares e hilos diamantados.

En una segunda parte de este trabajo, que aparecerá en un próximo número de esta revista, se analizarán las relaciones existentes entre las características intrínsecas de las rocas (básicamente textura, mineralogía y espacios vacíos), y su cortabilidad. Este tipo de estudios, es decir la interpretación de las propiedades de las rocas y la predicción de su comportamiento en función de sus características petrográficas, viene dando interesantes resultados en diversos campos de la Petrología aplicada $(1,2)$.

\section{INFLUENCIA DE LAS CARACTERÍSTICAS DE LA SIERRA DE CORTE}

Una de las características a considerar en el corte de rocas es la versatilidad de la máquina de corte, especialmente respecto a la posibilidad de variación de la potencia y la velocidad periférica del disco durante el proceso de corte. Hay que tener en cuenta que el corte de un material requiere un consumo de energía por parte de la sierra, de modo que, en materiales duros y para regímenes de corte altos -definiendo el régimen o velocidad de corte como la superficie de roca cortada por unidad de tiempo-, la potencia requerida puede llegar a ser superior a la suministrada. En estos casos, y si se quiere mantener el régimen de corte, se puede aumentar la velocidad periférica del disco, reduciendo de esta manera la potencia consumida.

Los problemas surgen cuando no existe la posibilidad de cambiar la velocidad de giro del disco, en cuyo caso, la única solución consiste en aumentar o disminuir el diámetro del disco diamantado (aumentando o disminuyendo respectivamente la velocidad periférica del disco), con la consiguiente modificación de la profundidad posible de corte.

El consumo de energía de la máquina (para un mismo régimen de corte) también se puede reducir disminuyendo la profundidad de corte y aumentando la velocidad transversal del disco (siendo ésta la velocidad de desplazamiento del disco respecto a la roca cortada). Además, cuanto mayor sea el consumo de energía, en general requerirá un mayor caudal de refrigerante.

El refrigerante es otro de los factores que hay que tener en cuenta. Su misión consiste en enfriar (especialmente el diamante), lubricar (reduciendo la fricción entre el diamante y la superficie de la roca), lavar (eliminando las esquirlas y lodos producidos en el corte de la roca) y facilitar la precipitación de estos lodos. La efectividad del tipo de refrigerante va a depender de la composición de los segmentos, de la concentración de diamante e incluso de las características de la roca a cortar. En este sentido, una selección adecuada del refrigerante puede aumentar la vida del disco y reducir la potencia consumida y el ruido producido durante el corte (3).

\section{IMPORTANCIA DEL DISCO DE CORTE}

El disco está constituido por un núcleo metálico o alma y una corona o segmento, formado por una matriz ligante metálica (generalmente de bronce o cobalto y a veces con diversas proporciones de wolframio y niquel) que engloba pequeños cristales de diamante.

Existen tres tipos fundamentales de discos diamantados: los de borde continuo (poco utilizados en los aserraderos de rocas ornamentales), los de segmentos con ranuras normales (los más utilizados para mármoles, granitos y pizarras) y los de segmentos con ranuras reducidas (que permiten un mejor acabado superficial aunque una menor velocidad de corte que los de ranuras normales) (4).

Son todos estos componentes, así como su diseño(diámetro, morfología de los segmentos o corona, tamaño del diamante, etc.) los que condicionarán la adecuación de un determinado disco al corte de los diversos tipos de materiales.

\subsection{El núcleo}

Es metálico (normalmente acero) y los principales requisitos que se le exigen son que no se desgaste facilmente y que no alabee durante el proceso de corte.

\subsection{EI diamante}

En primer lugar hay que considerar el tipo de diamante, ya que la naturaleza y forma de los diamantes puede variar, afectando también a las características de corte. Por una parte están los diamantes sintéticos, los más utilizados, ya que permiten un condicionamiento de su forma y tamaño durante el proceso de producción a la vez que una calidad más uniforme, $y$, por otra parte, están los naturales, con formas más irregulares, pero que poseen una mejor 
retención en la matriz, aristas de corte más agudas y mayor estabilidad térmica (5).

La selección del tipo de diamante está en función de la cortabilidad de la roca a procesar $(6,7)$.

Otra característica a considerar es el tamaño de grano del diamante, que suele oscilar entre 1 y $0,2 \mathrm{~mm}$. En general, los tamaños mayores se utilizan para las rocas más blandas, ya que éstas permiten un régimen de corte mayor, por lo que la eliminación de las partículas minerales liberadas durante el corte tiene que ser también rápida. El mayor tamaño del diamante permite un mayor espacio entre la matriz ligante y la roca, facilitando el lavado y arrastre de las partículas.

Del mismo modo, existe una relación entre el tamaño de grano del diamante, la velocidad periférica del disco y la velocidad o régimen de corte: si se mantiene constante la velocidad periférica del disco y se aumenta la velocidad de corte, se requiere un tamaño mayor del diamante, para facilitar la eliminación del polvo de roca, como en el caso anterior (5).

En rocas duras se recomiendan diamantes con tamaño de grano fino, ya que los cristales pequeños ofrecen una mayor resistencia a la compresión y al choque (7).

La concentración del diamante en la matriz es otro aspecto a considerar. En general, se puede decir que cuanto mayor sea esta concentración, mayor es la vida del disco, aunque, normalmente, también va a ser mayor su precio. Además, hay que tener en cuenta que una mayor concentración de diamante requiere un mayor consumo de potencia de la máquina, debido al aumento de las fuerzas de rozamiento entre el disco y la roca.

\subsection{La matriz}

La naturaleza de la matriz que engloba a los diamantes y especialmente su dureza reviste una cierta importancia. Para conseguir un buen rendimiento del disco es necesario que, aun siendo muy diferente la dureza del diamante y de la matriz, se desgasten ambos simultáneamente, de modo que los diamantes tengan una exposición constante y suficiente. Si la matriz es demasiado dura, los diamantes no estarán suficientemente en relieve para cortar con eficacia (por lo que sería conveniente "afilar" el disco cortando una roca o material más abrasivo), mientras que si la matriz es demasiado blanda, los diamantes se "descalzan" demasiado pronto y se desprenderán fácilmente (8).

\subsection{Procesos de desgaste del disco}

Durante el proceso de corte, el disco gira siempre en el mismo sentido produciéndose, al desgastarse la matriz ligante, el afloramiento de los cristales de diamante de los segmentos. Esto hace que se produzca una "cola" de matriz detrás de cada cristal de diamante que lo fija durante el corte, evitando que se despegue (Fig. 1). Esto no sucede cuando se utiliza un telar diamantado, ya que, al desplazarse en dos direcciones opuestas, la "cola" no se forma, desprendiéndose los diamantes con mayor facilidad (por eso, cuando se quita un disco diamantado hay que volver a ponerlo en la sierra en la misma posición anterior). De aquí también la conveniencia de utilizar en los telares diamantados cristales de diamante con formas irregulares, que posean una mayor adherencia con la matriz.

El despegue de los cristales de diamante (y, por tanto, el desgaste de la matriz debido a la abrasividad de las rocas)

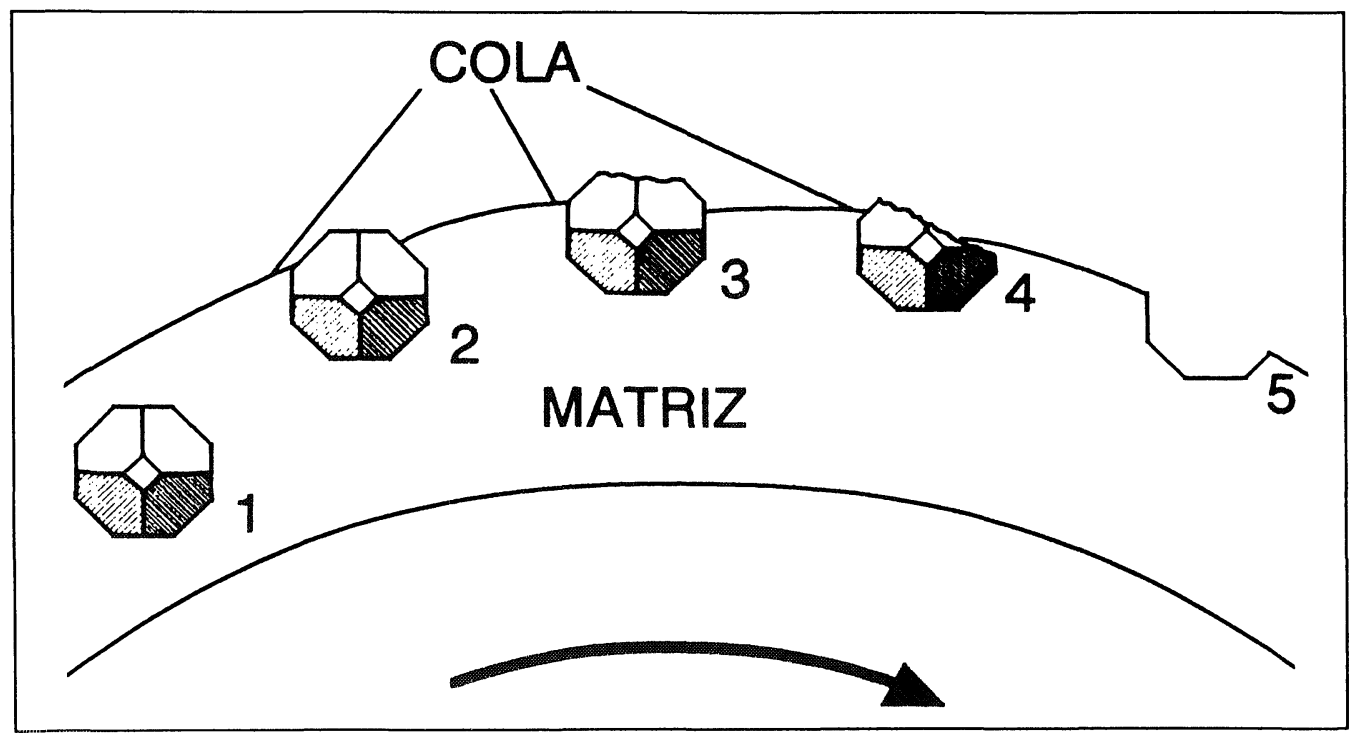

Fig. 1.- Fases por las que pasan los cristales de diamante durante el corte: 1) incluidos en la matriz; 2) inicio de la actividad; 3) comienzo del desgaste; 4) fracturación y 5) despegue o saltación (8). 
es uno de los mecanismos de desgaste del disco, pero hay que destacar otros procesos de rotura y pulido del diamante que contribuyen a reducir la vida útil del disco.

Cuando se corta una roca con disco diamantado, se genera en el disco (y, por tanto, en los diamantes de los segmentos) unos esfuerzos compresivos y otros de cizalla que hacen que los cristales de diamante se rompan o pulan. Los esfuerzos compresivos dependen de algunos parámetros de corte, como la profundidad del corte, sentido de giro del disco respecto a la superficie cortada, etc., mientras que los de cizalla están más condicionados por la velocidad transversal o de avance del disco. McGowan y Brauninger (9), mediante la observación de los diamantes de la superficie del disco, explican los mecanismos de desgaste en función de los parámetros de trabajo y, por tanto, de las fuerzas que actúan sobre el disco (Fig. 2). En la tabla I se esquematizan las condiciones de corte, los fenómenos de

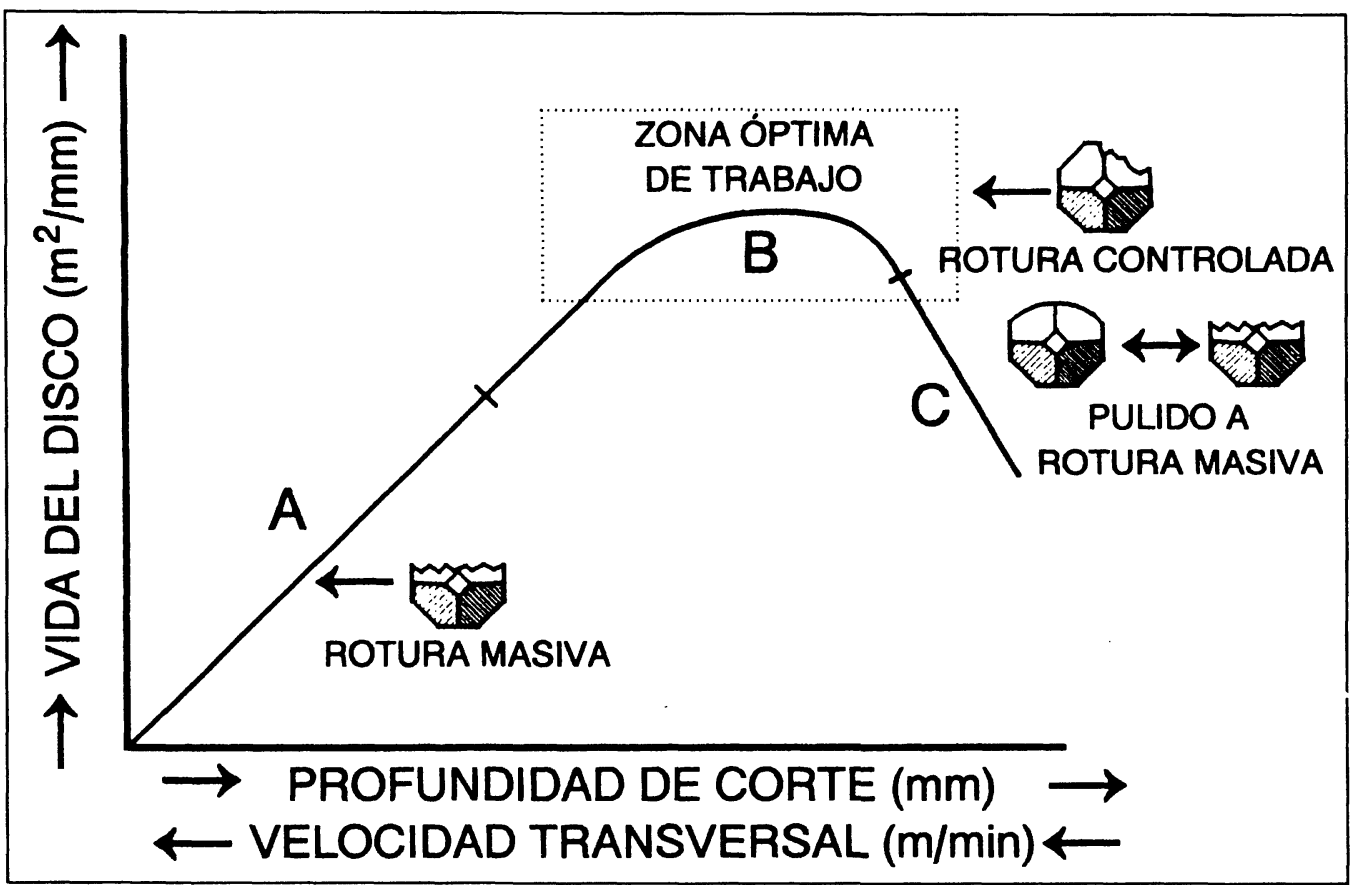

Fig. 2.- Vida útil del disco ( $\mathrm{m}^{2}$ cortados por $\mathrm{mm}$ de desgaste del diámetro) en función de la velocidad transversal y de la profundidad de corte (para régimen de corte constante) (9).

TABLA I

Características del corte y rendimiento del disco en función de la carga y deformación de los cristales de diamante (9)

\begin{tabular}{||c|c|c||}
\hline $\begin{array}{c}\text { Carga elevada } \\
\text { (Rotura A) }\end{array}$ & $\begin{array}{c}\text { Carga adecuada } \\
\text { (Rotura B) }\end{array}$ & $\begin{array}{c}\text { Carga insuficiente } \\
\text { (Rotura C) }\end{array}$ \\
\hline $\begin{array}{c}\text { Fracturación masiva } \\
\text { del diamante }\end{array}$ & $\begin{array}{c}\text { Microfracturación } \\
\text { controlada del diamante }\end{array}$ & $\begin{array}{c}\text { Pulido o rotura masiva } \\
\text { del diamante }\end{array}$ \\
\hline $\begin{array}{c}\text { Reducida vida } \\
\text { útil }\end{array}$ & $\begin{array}{c}\text { Óptima vida } \\
\text { útil }\end{array}$ & $\begin{array}{c}\text { Paulatina disminución } \\
\text { de la vida útil }\end{array}$ \\
\hline $\begin{array}{c}\text { Desviación del } \\
\text { corte }\end{array}$ & $\begin{array}{c}\text { Buena calidad del } \\
\text { corte }\end{array}$ & $\begin{array}{c}\text { Desviación del } \\
\text { corte }\end{array}$ \\
\hline $\begin{array}{c}\text { Gran consumo de } \\
\text { potencia }\end{array}$ & $\begin{array}{c}\text { Reducido consumo } \\
\text { de potencia }\end{array}$ & $\begin{array}{c}\text { Creciente consumo de } \\
\text { potencia }\end{array}$ \\
\hline & $\begin{array}{c}\text { Bajo nivel de } \\
\text { ruido }\end{array}$ & $\begin{array}{c}\text { Baja velocidad de } \\
\text { corte }\end{array}$ \\
\hline
\end{tabular}


desgaste de los diamantes y las consecuencias derivadas sobre el rendimiento del corte.

También Ertingshausen (10) explica los procesos de desgaste del disco: cuando las fuerzas de corte son elevadas se rompen los cristales de diamante, produciendo nuevas aristas cortantes y reduciendo la profundidad de corte del diamante. Después de que rompa varias veces el diamante, se desgasta la matriz y salta el diamante. Por el contrario, cuando las fuerzas de corte son pequeñas (o cuando se trata de una roca muy abrasiva) las aristas del diamante se redondean progresivamente, hasta que el cristal se desprende de la matriz.

Así pues, la rotura de los diamantes debe ser la suficiente para "autoafilarse" el borde; la saltación o arranque de diamantes debe evitarse o retrasarse lo más posible y el desgaste o pulido, producido por la transformación en grafito de la superficie del diamante debido al calentamiento, debe suprimirse aumentando la refrigeración o utilizando velocidades de corte más elevadas (11).

\section{INCIDENCIA DE LOS PARÁMETROS DEL CORTE}

El modo de corte, referido al sentido de giro del disco respecto a la dirección de corte del material, es un factor que afecta a la distribución de fuerzas en el disco ( $y$, por tanto, a su rendimiento) así como al tipo de desgaste que sufre. Cuando el sentido de giro coincide con el de desplazamiento relativo del material a cortar ("downcutting") se generan mayores fuerzas verticales (compresivas) y menores fuerzas horizontales (de cizalla) que cuando el sentido de giro es al contrario ("up-cutting").
Por tanto, las características de desgaste del disco variarán (Fig. 3).

Ertingshausen (10), estudiando el granito Rojo Colombo, observó que, con el "up-cutting", el desgaste de la periferia del disco de corte era aproximadamente uniforme, mientras que con el "down-cutting", el desgaste era cíclico y progresaba en la dirección opuesta a la de giro del disco. Para pequeñas profundidades de corte, el "down-cutting" desgasta más el disco, pero a partir de $25-30 \mathrm{~mm}$ de profundidad, sucede al revés. Esto indica que el modo de corte elegido nos condicionará la profundidad de corte, si queremos obtener un buen rendimiento del disco. No obstante, el desigual desgaste de la periferia del disco durante el "down-cutting" puede llegar a producir importantes vibraciones cuando se cortan profundidades grandes.

En las rocas más blandas suele ser posible alcanzar grandes profundidades de corte, con lo que muchas veces se puede cortar la pieza de un solo corte ("single-pass cutting"). Sin embargo, en las rocas duras, esto no suele ser posible y hay que realizar varias "pasadas", generalmente alternando la dirección de movimiento del disco respecto a la roca ("step-cutting"), con lo cual también se alternan el "downcutting" y el "up-cutting". Esta alternancia suele disminuir la vida útil del disco (10).

Otro de los parámetros de corte es el régimen o velocidad de corte (expresado normalmente en $\mathrm{cm}^{2} / \mathrm{min} \mathrm{o} \mathrm{m}^{2} /$ hora), que dependerá de la profundidad del corte y de la velocidad transversal relativa del disco respecto a la roca. Para cualquier roca, manteniendo un régimen de corte constante, es mejor utilizar una velocidad transversal más

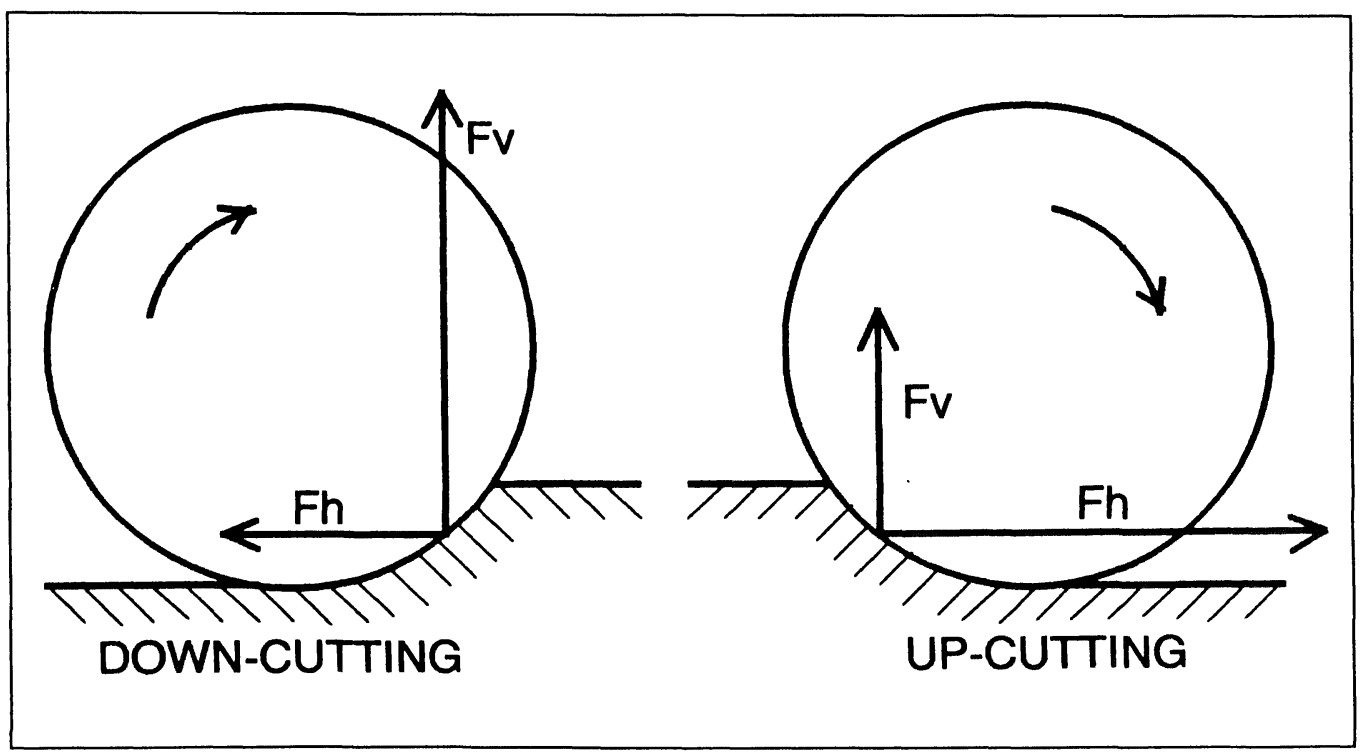

Fig. 3.- Representación gráfica de las fuerzas horizontales $(F h)$ y verticales (Fv) durante el corte hacia abajo (down-cutting) y hacia arriba (up-cutting). 
baja asociada a una mayor profundidad de corte para aumentar el rendimiento del disco. Un aumento del régimen de corte conlleva, en general, una disminución de la vida útil del disco (Fig. 4) y un aumento de la energía consumida y del agua de refrigeración $(12,5)$.

En general, los granitos duros se cortan a regimenes decorte lentos, del orden de $100 \mathrm{~cm}^{2} / \mathrm{min}$, y los granitos más blandos a mayores regímenes (alrededor de $1.000 \mathrm{~cm}^{2} / \mathrm{min}$ ). De todos modos, para cada tipo de disco y para cada tipo de granito existe una velocidad de corte más económica que hay que determinar, para conseguir un mejor rendimiento del proceso de corte (12).

El régimen de corte, junto con el tipo de material a cortar, condicionan la velocidad periférica óptima del disco. En general esta velocidad es menor para el corte de granitos duros (del orden de $25-30 \mathrm{~m} / \mathrm{s}$ ) que de granitos blandos $(35-40 \mathrm{~m} / \mathrm{s})$ y, a su vez, menor que el de otros materiales más blandos, como areniscas porosas (alrededor de $60 \mathrm{~m} / \mathrm{s})(12, \mathrm{~s})$.

Burgess y Birle (12) han hallado, para el granito de Barre (roca de cortabilidad media), que para cada régimen de corte existe una velocidad periférica óptima del disco
(Fig. 4); existe una relación directa entre ambas y asi, al aumentar el régimen de corte, la velocidad periférica adecuada para obtener un mayor rendimiento del disco también aumenta.

No obstante, en la práctica, la selección definitiva de una velocidad periférica óptima del disco está influenciada por otros factores, como el diseño del disco, concentración de diamante, naturaleza de la matriz, etc. (12).

\section{IMPORTANCYA DEL FACTOR HUMANO}

Como por lo general la máquina suele ser una "constante" en las naves de elaboración de las rocas ornamentales (es decir, que normalmente no se renuevan cada poco tiempo), al menos drben mantenerse en buen estado para obtener los mejores resultados posibles. En este sentido tiene gran importancia el factor humano en la detección de incipientes fallos de la máquina y la corrección de ellos, en especial la eliminación de las vibraciones por mal estado de los rodamientos.

El operador es, en ocasiones, el factor más importante para un buen rendimiento del corte con útiles diamantados. Sin duda una formación profesional especializada o cursos de

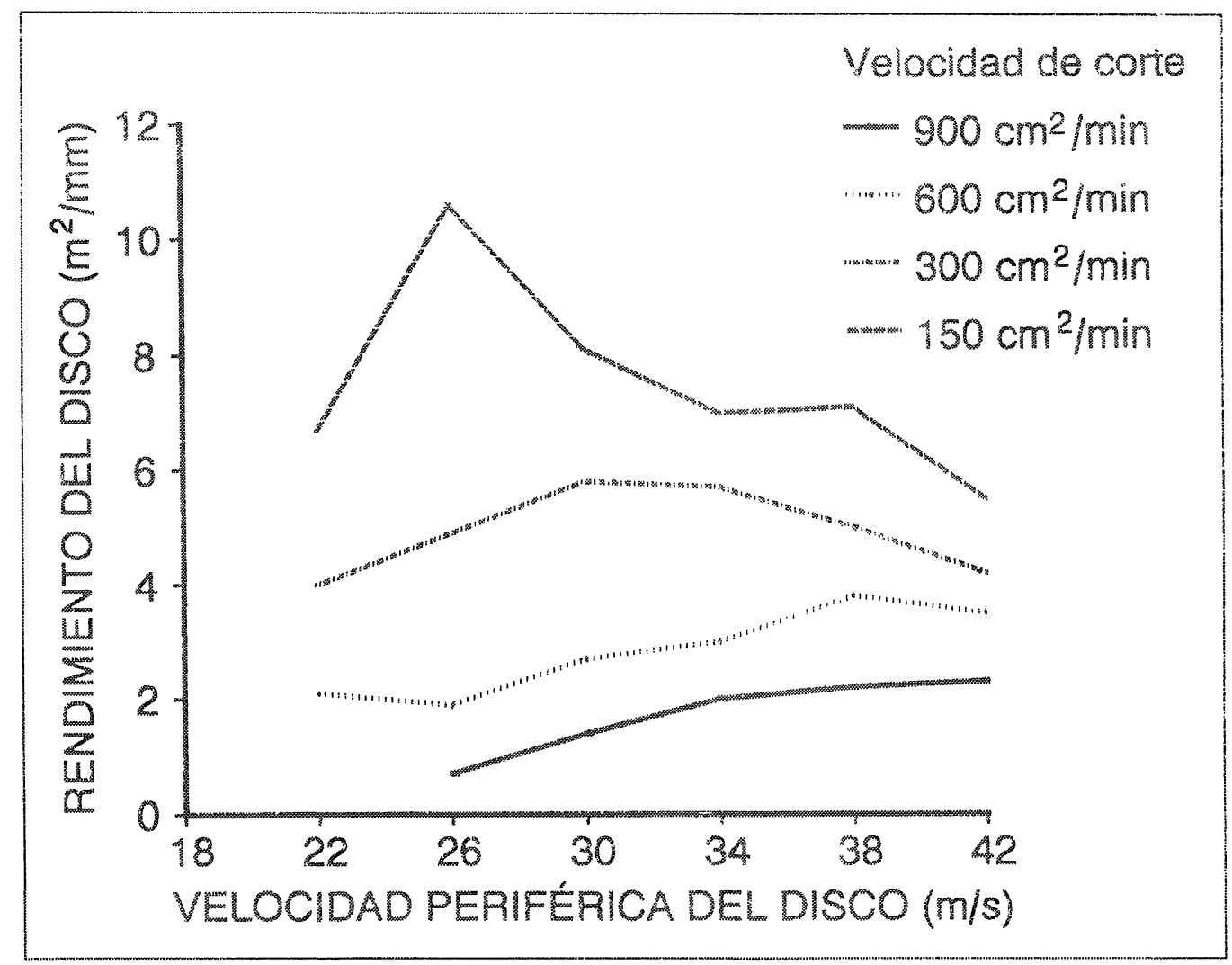

Fig. 4.- Rendimicnto del disco $\left(\mathrm{m}^{2}\right.$ cortados por mm de desgaste del diámetro) frente a velocidad periférica óptima para distintas velocidades de curte (granito de Barre) (12). 
manejo y perfeccionamiento de las técnicas de corte pueden contribuir a reducir los gastos globales del corte de rocas.

\section{CONCLUSIONES}

El rendimiento del corte de rocas con discos diamantados está condicionado por una serie de parámetros propios del sistema de corte. La selección de una sierra versátil (que permita modificar tanto la potencia como la velocidad periférica del disco y su velocidad transversal), lautilización de un disco diamantado adecuado para las características de la roca a cortar y el establecimiento y empleo de las condiciones idóneas de corte, así como una formación adecuada del operador, permitirán reducir gastos en la etapa de corte de rocas ornamentales y mejorar la calidad del acabado de las superficies cortadas.

\section{AGRADECIMIENTOS}

A la Universidad de Oviedo, por la subvención SV-VIC95-0027 concedida para la realización de este trabajo.

\section{BIBLIOGRAFÍA}

(1) CALLEJA, L.; M. MONTOTO; L.M. SUÁREZ DEL RÍO Y A. RODRÍGUEZ REY (1982). "Relaciones entre petrografía, grado de alteración y resistencia a la compresión en rocas graníticas". Obras de superficie en mecánica de rocas. Soc. Esp. de Mecánica de Rocas (Ed.) Vol. 1, Cap. 1-1, 12 pp.

(2) ESBERT, R.M.; R. MARCOS; J. ORDAZ; M. MONTOTO; L.M. SUÁREZ DEL RÍO; V.G. RUIZ DE ARGANDOÑA; L. CALLEJA; F.J. ALONSO Y A. RODRÍGUEZ REY (1989).- "Petrografia, propiedades físicas y durabilidad de algunas rocas utilizadas en el patrimonio monumental de Catalunya, España". Materiales de Construcción, V. 39, n 214, pp 37-47.

(3) WANG, C.Y.; X. WEY; Z.L. TANG Y Z.C. PAN (1995).- "The role of coolant in granite sawing". Industrial Diamond Review, 4/95, pp. $156-160$.

(4) ZUAZO TORRES, P. (1995).- "Elaboración de mármoles y granitos". En "Manual de Rocas Ornamentales. Prospección, explotación, elaboración y colocación". Carlos López Jimeno (Ed.), Entorno Gráfico, S.L., Madrid, 696 pp.

(5) JENNINGS, M. Y D. WRIGHT (1989).- “Guidelines for sawing stone”. Industrial Diamond Review, 2/89, pp. 70-75.

(6) WRIGHT, D.N. Y V.B. CASSAPI (1985).- “Factors influencing stone sawability”. Industrial Diamond Review, $2 / 85$, pp. 84-87.

(7) PERRIER, R. (1993 a).- "Le diamant dans le sciage circulaire des roches. 2ème partie". Le Mausolée, 4/93, pp. 40-45.

(8) PERRIER, R. (1993 b).- “Le diamant dans le sciage circulaire des roches. 3ème partie”. Le Mausolée, 5/93, pp. 44-49.

(9) McGOWAN, J. Y G. BRAUNINGER (1991).- “The application of supperabrasives in granite slabbing”. Superabrasives'91. Junio, 11-13, 1991. Chicago, Illinois (Existe una traducción de este trabajo en Roc Máquina, Nov. 91, pp. 97-110).

(10) ERTINGSHAUSEN, W. (1985).- "Wear processes in sawing hard stone”. Industrial Diamond Review, 5/85, pp. $254-258$.

(11) BORTOLUSSI, A.; A. CARANASSIOS; R. CICCU; R. LASSANDRO; P.P. MANCA Y G. MASSACCI (1992).- "Progress in the knowledge of granite cutting with diamond wire". 11 th Int. Conf. on Ground Control in Mining, The University of Wollongong, N.S.W., pp. 593-599.

(12) BURGESS, R.R. Y J.D. BIRLE (1978).- "El corte de granito con discos de diamante". 30 Asamblea Seminario de la Asociación de Diamante Industrial de Japón. Tokyo. Existe versión en castellano aparecida en Roc-Máquina: Burgess, R.R. (1989). "El corte de granito con disco de diamante". Roc-Máquina, $3^{\text {er }}$ trimestre de 1989, pp. 22-35. 